\title{
Acoustic Virtual Vortices with Tunable Orbital Angular Momentum for Trapping of Mie Particles
}

\author{
Asier Marzo, ${ }^{*}$ Mihai Caleap, and Bruce W. Drinkwater \\ Department of Mechanical Engineering, University of Bristol. University Walk, Bristol BS8 1TR, United Kingdom
}

(Received 27 June 2017; published 22 January 2018)

\begin{abstract}
Acoustic vortices can transfer angular momentum and trap particles. Here, we show that particles trapped in airborne acoustic vortices orbit at high speeds, leading to dynamic instability and ejection. We demonstrate stable trapping inside acoustic vortices by generating sequences of short-pulsed vortices of equal helicity but opposite chirality. This produces a "virtual vortex" with an orbital angular momentum that can be tuned independently of the trapping force. We use this method to adjust the rotational speed of particles inside a vortex beam and, for the first time, create three-dimensional acoustics traps for particles of wavelength order (i.e., Mie particles).
\end{abstract}

DOI: 10.1103/PhysRevLett.120.044301

Acoustic vortices have been extensively studied for their ability to trap particles [1-3] and transfer orbital angular momentum (OAM) [4-13]. Acoustic vortices have been theorized to generate negative radiation forces along the propagation direction $[1,14,15]$, and recently, the stable three-dimensional trapping of Rayleigh particles (i.e., radius smaller than the wavelength $a \ll \lambda$ ) was demonstrated using focused acoustic vortices $[16,17]$. We note that, in this context, a stable trap is one in which the forces are convergent on the particle and that the system is relatively insensitive to small perturbations.

Acoustic vortices are wave fields with a screw dislocation in the phase wave front [18] and an amplitude null on the axis

$$
p_{\text {in }}=P(r, \theta) e^{i \ell \varphi} e^{-i \omega t},
$$

where $(r, \theta)$ are polar coordinates, $\omega$ is angular frequency, and $P(r, \theta)$ is an axisymmetric field, typically a first or higher order Bessel function, that satisfies the scalar wave equation. The phase factor $\exp (i \ell \varphi)$ describes the helicoidal motion around the propagation axis ( $\varphi$ refers to the azimuth angle), and $\ell$ defines the helicity (or topological charge); its sign defines the handedness, or chirality, of the wave front propagation.

In an acoustic vortex, both the trapping force and the OAM are proportional to the total power of the beam [10-12], meaning that the rotational speed cannot be controlled independently of the trapping force. This coupling results in dynamic instability; e.g., when high powers are needed to create stiff traps, the by-product is high OAM, resulting in instability and possible ejection of the levitated particles [16].

Published by the American Physical Society under the terms of the Creative Commons Attribution 4.0 International license. Further distribution of this work must maintain attribution to the author(s) and the published article's title, journal citation, and DOI.
Acoustic methods have distinct advantages over other levitation methods, for example, optical, magnetic, or electrostatic levitation, particularly in their ability to levitate a wider range of materials in different host fluids. Consequently, acoustic trapping is a fundamental tool in chemistry [19], blood analysis [20], the study of organisms in microgravity $[21,22]$, control of nanomaterial self-assembly [23], and x-ray crystallography [24]. At the same time, contactless rotation has proven invaluable for understanding planetary formation [25] and acoustic streaming has been used to rotate microorganisms for microscopy [26]. If the link between OAM and trapping force could be broken, acoustic levitation could become an affordable and adaptable method for contactless rotation of samples due to the variety of supported materials and high efficiency.

It has been shown that an acoustic twin trap can be used to rotate a levitated particle $[16,27]$ by altering the orientation of the high pressure "fingers." However, this approach required the use of asymmetric particles and the speed of rotation was limited to the phase refresh rate of the drive electronics and emitting devices. Acoustic vortices of fractional order [28] and short-pulsed vortices [29] have noninteger topological charges, but the OAM is still coupled to the beam power and trapping force. In optics, the polarization of the two beams that make a vortex can be adjusted to change the amount of OAM independently from the trapping strength [30]; however, as sound is a scalar field, this approach cannot be used directly in acoustics.

Here, we show that rapidly time-multiplexed acoustic vortices of opposite direction create a time-averaged vortex, or virtual vortex, which can have trapping forces and OAM that are independently tunable. Acoustic virtual vortices are shown to be able to stably trap and effectively control the rotational speeds of levitated samples. Additionally, a virtual vortex of large aperture (i.e., high helicity) is shown to steadily trap particles with diameters 
larger than the wavelength-a result which surpasses the classical Rayleigh scattering limit that has previously restricted stable acoustic particle trapping.

To explore these concepts experimentally, two multielement arrays have been constructed using air-coupled ultrasound transducers operating at $40 \mathrm{kHz}$ (wavelength $\lambda=8.66 \mathrm{~mm}$ in air at $25^{\circ} \mathrm{C}$ ). These arrays are named the spherical cap (SC) and open spherical sector (OSS). The SC array has a diameter of $110 \mathrm{~mm}$, geometrical focus of $70 \mathrm{~mm}$, and is made of 52 air-coupled transducers (See Supplemental Material Fig. S1 in Ref. [31]); we used this array to trap expanded polystyrene particles (EPS), with diameters ranging from 0.5 to $5 \mathrm{~mm}$. The OSS array has an $f$ number (focal length to aperture) equal to 0.1 , with an outer diameter of $195 \mathrm{~mm}$ and an inner diameter of $120 \mathrm{~mm}$. It is made of 192 transducers (Fig. S2 in Ref. [31]); this array is used to trap EPS particles of up to $16 \mathrm{~mm}$ in diameter, corresponding to $\sim 1.85 \lambda$.

Through a series of experiments and accompanying numerical simulations, we now demonstrate (a) the inherent instability of airborne acoustic vortices, (b) stabilization of a vortex beam using the virtual vortices concept, (c) control of rotational speeds of levitated samples, and finally, (c) levitation of particles with diameters of wavelength order (i.e., Mie particles).

Here, we term an acoustic vortex defined by Eq. (1) a regular vortex $(\mathrm{RV})$. Such vortices have been previously shown to be suitable for trapping and manipulating Rayleigh particles. However, as the particles get larger, they start to orbit around the vortex core until being ejected out of the trap. This dynamic behavior is observed both in simulations and experiments, as shown in Figs. 1 and 2 as well as in the Supplemental Material (Movie S1 in Ref. [31]). The inability of acoustic vortices to trap particles larger than the first high-intensity ring has been hinted at before [44], but the actual limit in air is much lower. In principle, one might expect that a particle smaller than the first high-intensity ring may be stably trapped (i.e., $\sim 6 \mathrm{~mm}$ in our setup for a regular vortex with helicity $\ell=1$ ). However, in experiments, even particles of $1.6 \mathrm{~mm}$ diameter are ejected. As the particle size increases, the tangential component of the exerted force field increases, which causes the particle to orbit around the beam axis during the trapping process. When the tangential force component cannot be sufficiently damped by the air drag, the orbit diameter increases until the particle is ejected. This leads to a threshold in the particle sizes that can be trapped (Fig. 2). This phenomenon has been theoretically predicted for particles trapped in optical vortices [45-47].

To make vortices stable for a wider range of particle sizes, we switch the emitted vortex chirality between $+\ell$ and $-\ell$ at high frequencies. Experimentally, this is achieved by switching the driver phases to make the array emit two different pressure fields. These pressure fields will each exert a time-averaged radiation force on the particle. The total effect on the particle is a combination of these different force fields. This idea builds on the concept of mode switching for controlling the height of particles passing through a standing wave in a microfluidic channel [48] and, in general, emerges from the acoustic radiation

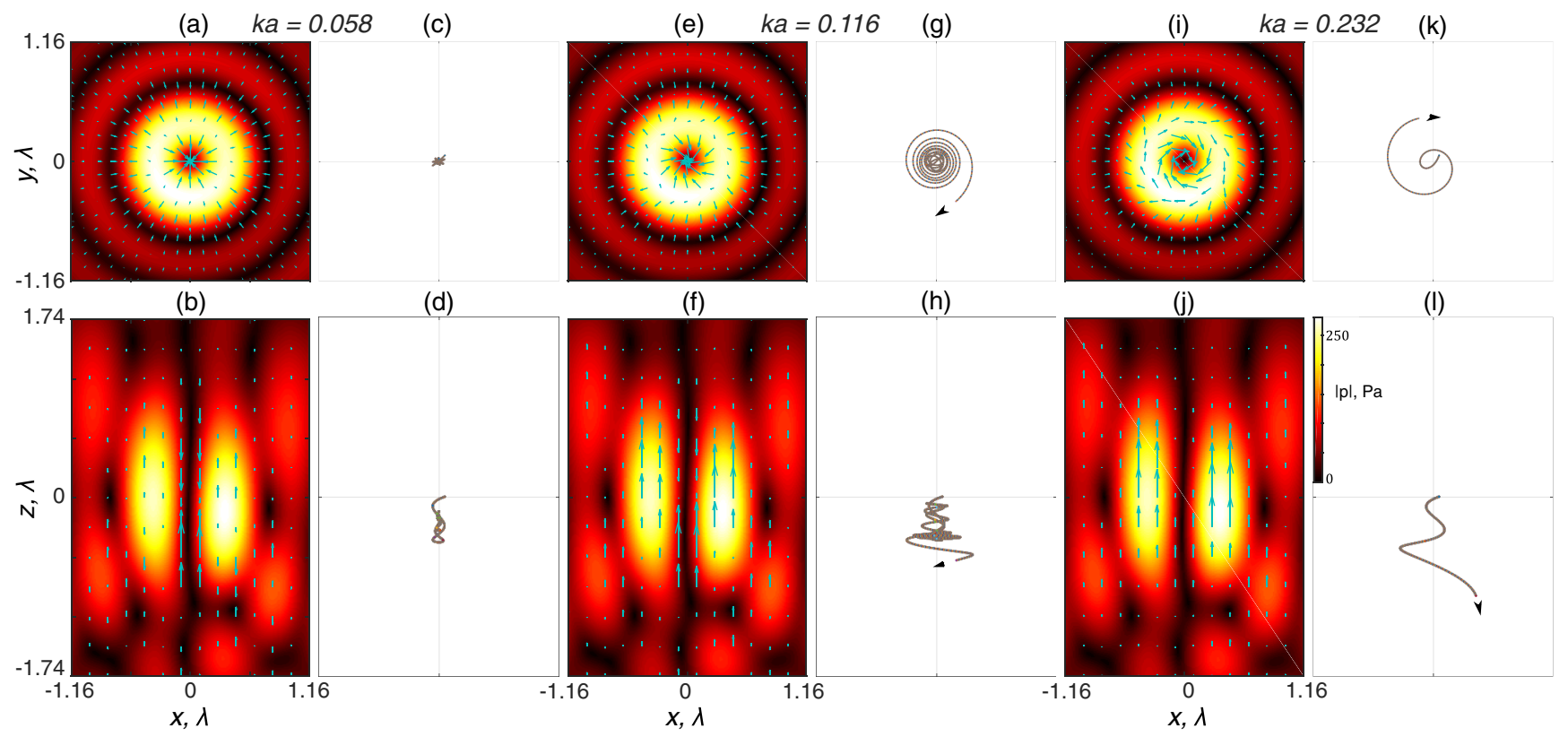

FIG. 1. Simulated amplitude fields of a RV1 (topological charge = 1) generated with the SC array using $15 \mathrm{Vpp}(\mathrm{a}),(\mathrm{b}),(\mathrm{e}),(\mathrm{f}),(\mathrm{i}),(\mathrm{j})$ ). Arrows represent the simulated forces $(z$ component not to scale) exerted on particles of radius $0.5 \mathrm{~mm}=0.058 \lambda(\mathrm{a}),(\mathrm{b}), 1 \mathrm{~mm}=$ $0.116 \lambda(\mathrm{e}),(\mathrm{f})$, and $4 \mathrm{~mm}=0.232 \lambda$ (i),(j). Simulated trajectories that the particles follow (c),(d),(g),(h),(k),(l). The $0.058 \lambda$ particle remains trapped, whereas the others get ejected. Axes and radius of the particles are in wavelengths $(\lambda),(k=$ wave number, $a=$ radius $)$. 


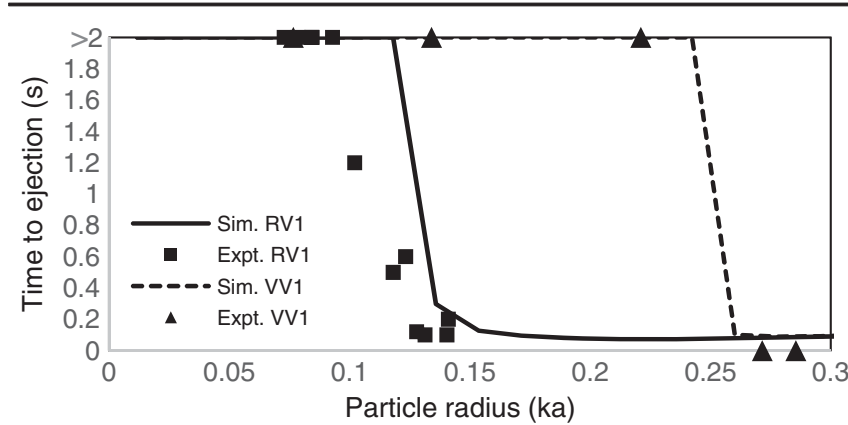

FIG. 2. Time to ejection as a function of the particle size for a RV1 and a VV1 generated with the SC array and an excitation signal of $15 \mathrm{Vpp}$. The particle is stably trapped after $2 \mathrm{~s}$. Radius of the particles are in wavelengths ( $k=$ wave number, $a=$ radius).

force being a time-averaged effect. Sequences of short vortices of opposite chirality have been generated before [49] and have been used for communications [50]. The idea of using them to control the rotational speed of particles has been hinted at before [51], but never modeled or realized experimentally.

A virtual vortex (VV), is formed by the combined effect of multiple, two in this case, RVs as

$$
\begin{aligned}
\left(\begin{array}{c}
\mathcal{F} \\
\mathcal{T}
\end{array}\right)_{\mathcal{V V}}= & \Pi^{+}\left(T N^{+}, T_{R}\right)\left(\begin{array}{l}
\mathcal{F}^{+} \\
\mathcal{T}^{+}
\end{array}\right)_{\mathrm{rad}} \\
& +\Pi^{-}\left(T N^{+}, T_{R}\right)\left(\begin{array}{c}
\mathcal{F}^{-} \\
\mathcal{T}^{-}
\end{array}\right)_{\mathrm{rad}}
\end{aligned}
$$

where

$$
\Pi^{+(-)}\left(T N^{+}, T_{R}\right)=\left\{\begin{array}{l}
1(0):\left(t+n T_{R}\right)<n T N^{+} \\
0(1):\left(t+n T_{R}\right) \geq n T N^{+}<n T_{R}
\end{array} .\right.
$$

Here, $\mathcal{F}_{\text {rad }}^{ \pm}$and $\mathcal{T}_{\text {rad }}^{ \pm}$are the force and torque fields due to the two component RVs. $\Pi^{+(-)}(t)$ describes a square wave of period $T_{R}$ that modulates the component RVs, such that only one exists at any moment in time. The switching point of this square wave is defined by $T N^{+}$, where $T$ is the period of the sinusoidal acoustic wave $(40 \mathrm{kHz})$ and $N^{+}$is the number of cycles of the first component. As can be seen, in general, the resulting force is a function of time and a timing diagram can be seen in the Supplemental Material (Fig. S3 in Ref. [31]). However, if the switching period $T_{R}$ is much longer than the period $T$ of the acoustic wave and much shorter than the period associated with the system's time constant $\tau$ (see Methods in Ref. [31]), then the particle can be thought of as being acted on by the overall time-averaged force.

In this study, we found that the fastest switching rate for generating a virtual vortex beam with our experimental parameters, without altering the stiffness of the trap, was $N^{+}=N^{-}=40$ (Fig. S4 in Ref. [31]). This corresponds to a constant modulation time $t_{M}^{ \pm}=T_{R} / 2=1 \mathrm{~ms}$ at $40 \mathrm{kHz}$ in air.

Figure 2 shows that VVs can trap a wider range of particle sizes compared to RVs. Our experiments indicate that particles with diameters of up to $4 \mathrm{~mm}$ can be stably trapped in $\operatorname{VV} 1(\ell= \pm 1)$, compared to $1.6 \mathrm{~mm}$ in a $\mathrm{RV} 1$. However, this $4 \mathrm{~mm}$ size limit was caused by the maximum axial force ( $z$ direction) achievable in our devices, rather than by the underlying dynamics. If we neglect the $z$ component, our time domain dynamic simulations (Methods in Ref. [31]) suggest that the size stability limit for a VV1 will be $\approx 6 \mathrm{~mm}$ (Fig. S5 in Ref. [31]).

Trajectory plots for particles trapped inside a VV1 are shown in the Supplemental Material (Fig. S6 in Ref. [31]); it is observed that VVs reduce the particle orbiting behavior relative to RVs and hence lead to particle dynamics as if the azimuthal force components had been canceled.

By regulating the amount of time that each direction of the vortex is emitted, the angular velocity of the particle can be adjusted (Movie S2 in Ref. [31]). Figure 3(a) shows the angular velocity of a $1.2 \mathrm{~mm}$ diameter particle trapped in a VV1, as a function of the number of periods $N^{ \pm}$of the emitted component vortices. Figure 3(b) shows that the particle remains in a constant $z$ position despite rotational speed changes, thus demonstrating the OAM has been decoupled from the trapping force. Figure 3(c) shows that the particle angular velocity can also be adjusted by changing the amplitude of the emitted vortices. However, in this case, the trapping force is coupled to the OAM, as can be observed from changes in the $z$ position [Fig. 3(d)].

Particles with diameters significantly larger than the wavelength have been ultrasonically manipulated in two dimensions underwater using high $f$ number focused beams (so called microbeams) [52,53]. However, this phenomenon only occurs if the particle is partially acoustically transparent. In practice, this principle does not work in air, as even low-density materials (i.e., EPS) have significantly higher acoustic impedances than the
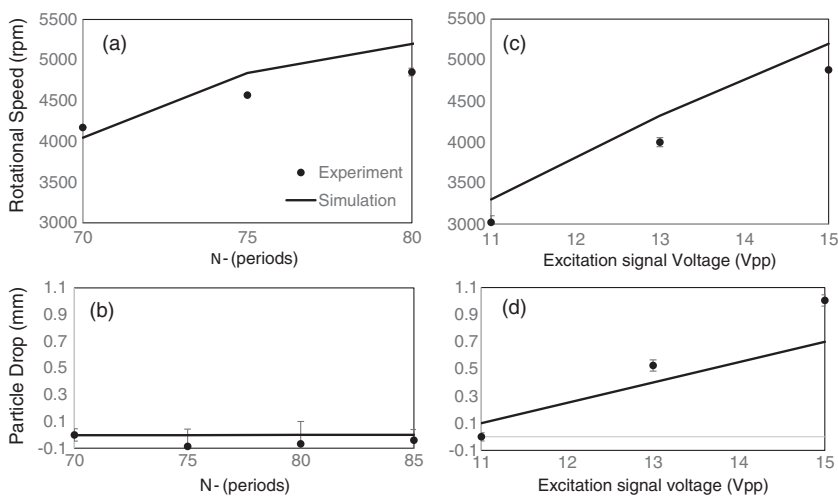

FIG. 3. Decoupling OAM from trapping force in a VV1. (a), (c) Angular velocity (in rpm) and (b),(d) axial drop against gravity of a $0.6 \mathrm{~mm}=(0.069 \lambda)$ radius particle as a function of (a),(b) number of cycles of $\boldsymbol{N}^{-}$(counterclockwise vortex) with clockwise vortex $\left(\boldsymbol{N}^{+}\right)=40$ periods and (b),(d) the amplitude of the excitation signal for a VV1 $\left(\boldsymbol{N}^{-}=80, \boldsymbol{N}^{+}=40\right)$. Experiments were repeated 5 times and the error bars represent the standard deviation. 
surrounding air. Alternatively, we note that large objects have been levitated using near field acoustic effects [54-56]; i.e., the sources must be placed around the object and in close proximity (on the order of a wavelength) to the surface of the object. However, despite the above progress, three-dimensional stable acoustic trapping of particles inside a standing wave or single beam has been fundamentally limited to particles smaller than half wavelength [57].

Controlling the aperture of the central vortex null by increasing the helicity of the vortex $(\ell>1)$ has been suggested as a method for trapping of Mie particles [58]. A RV of high helicity would not be able to trap such particles due to the previously shown instability, especially in this case, because the OAM is proportional to the helicity. The acoustic power of the beam can be reduced to reduce the OAM, but this also weakens the trapping force. However, using VVs of high helicity, wavelengthscale particles can be trapped as shown below and in the Supplemental Material (Movie S3 in Ref. [31]).

Here, we use the larger OSS array to generate VV3 and VV5. A $10 \mathrm{~mm}(1.18 \lambda)$ diameter EPS particle (weight $10 \mu \mathrm{g}$ ) was stably trapped using an excitation signal of $12.5 \pm 0.1 \mathrm{~V}$ in a VV3 (Fig. S7 in Ref. [31]). We note that the predictions of the axial force were $4.3 \%$ smaller than observed in the experiments. Furthermore, a particle of $16 \mathrm{~mm}(1.88 \lambda)$ diameter (weight $35 \mu \mathrm{g}$ ) was stably trapped using an excitation signal of $15.4 \pm 0.6 \mathrm{~V}$ in a VV5 (Fig. 4), and the predictions of the axial force were $56 \%$ smaller than the experiments. The poorer experiment-tosimulation agreement seen in the VV5 is thought to be due to surface irregularities in the hand-carved $16 \mathrm{~mm}$ diameter particle and the inaccuracies associated with dropping tests [59]. In Supplemental Material Movie S5 [31], the device is tilted beyond $90^{\circ}$, while a $10 \mathrm{~mm}(1.18 \lambda)$ diameter particle is trapped inside a VV3.

We have shown that regular airborne acoustic vortices are inherently unstable for trapping particles, even when they are smaller than half-wavelength diameter. This instability originates from the OAM that is partially transferred as a tangential force component that cannot be sufficiently damped and thus accumulates, increasing the orbit diameter. We have created virtual vortices by rapidly switching between component vortices of equal helicity and opposite chirality. By varying the proportions of these component vortices, the OAM can be tuned independently from the total beam power and trapping force, leading to stable trapping of wavelength order (i.e., Mie) particles. Fine rotational velocity control was achieved, as can be seen from the small error bars in Fig. 3(a) (1\% standard deviation over mean value).

To ensure stability of the virtual vortices, the switching rate must be tuned considering the dynamics of the system, which depends critically on particle size. To avoid ejection of EPS particles of $1.6 \mathrm{~mm}$ diameter, the switching speed
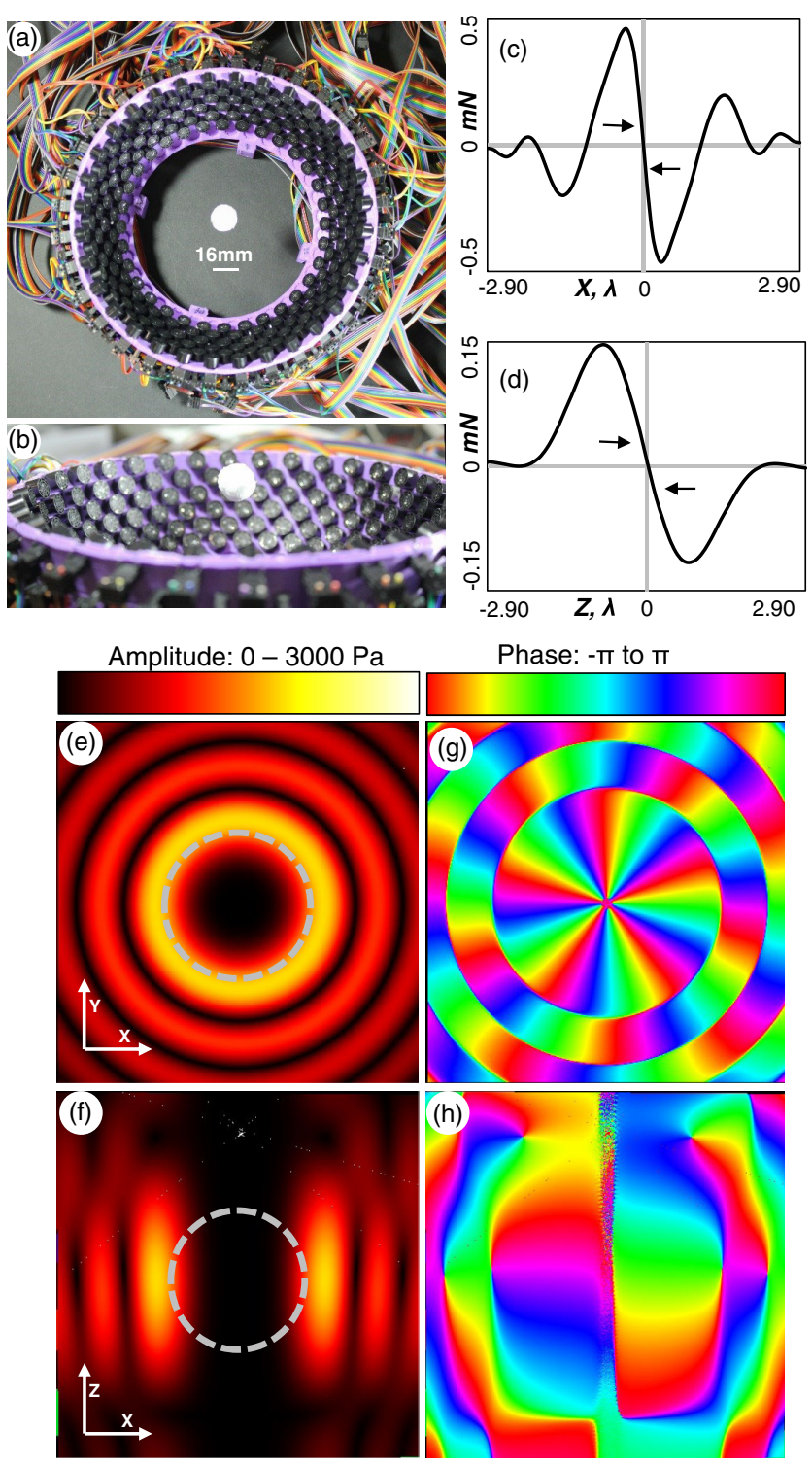

FIG. 4. (a),(b) An EPS particle of $16 \mathrm{~mm}=1.86 \lambda$ diameter trapped in a VV5 (topological charge $=5$ ) in the OSS array. (c) Simulated force along $X$ or $Y$ directions centered on the focus; horizontal axis is in terms of wavelengths $\lambda$. (d) Simulated forces along the $Z$ direction centered on the focus. Simulated amplitude fields in the $X Y$ (e) and $X Z$ (f) planes. Simulated phase fields in the $X Y(\mathrm{~g})$ and $X Z(\mathrm{~h})$ planes. The gray dashed circle in (e) and (f) represent the sphere.

needs to be faster than 1200 periods ( $30 \mathrm{~ms}$ ), for particles of $2 \mathrm{~mm} 5000$ periods $(13 \mathrm{~ms}$ ) and for particles of $3 \mathrm{~mm} 1000$ periods (2 ms) (Fig. S8 in Ref. [31]). Through analysis of the particle dynamics simulations, the effect that dominated here is that, as particle size increases, the tangential component of the force field also increases (see force vectors in Fig. 1).

For the first time, we have achieved stable acoustic 3D trapping of wavelength-scale particles $(16 \mathrm{~mm}$ or $1.88 \lambda)$. Using a more powerful device, our simulations suggest that, using virtual vortices, we could have reached particle 
sizes of up to $20 \mathrm{~mm}(2.32 \lambda)$ using a VV5 (Fig. S5 in Ref. [31]). In Supplemental Material Movie S4 [31], we show two-dimesional trapping of a particle of $5 \mathrm{~cm}$ diameter (5.88 $\lambda$ ), supported on a table, and control its rotation with a VV12.

All the presented results are for airborne acoustic vortices, but simulations (Fig. S9 in Ref. [31]) suggest that the instability due to accumulation of orbital momentum also affects vortices in other media, such as water or blood. Instabilities were reported when trapping particles in water in two dimensions using high-aperture vortices [58], although their cause was not certain.

We anticipate that virtual vortices will allow novel applications in particle centrifugation and manipulation of arbitrarily sized particles. With the standard acoustic levitation methods, numerous applications have been developed: i.e., crystallography [24], microscopy [60], and spectroscopy [61]. Therefore, the introduction of virtual vortices will extend the current uses of acoustic levitation to allow larger objects to be manipulated and their rotation controlled. Virtual vortices can also benefit other fields in which vortices are employed, such as microfluidics [62,63], optics [45], and electron beams [64].

This project has been funded by the U.K. Engineering and Physical Science Research Council (EP/N014197/1). All data needed to complete the study are contained within this paper.

* To whom all correspondence (inquiry) should be addressed. Department of Mechanical Engineering, University Walk, Clifton BS8 1TR, Bristol, United Kingdom. amarzo@hotmail.com; mihai.caleap@bristol.ac.uk

[1] D. Baresch, J.-L. Thomas, and R. Marchiano, J. Appl. Phys. 113, 184901 (2013).

[2] S. A. Seah, B. W. Drinkwater, T. Carter, R. Malkin, and S. Subramanian, IEEE Trans. Ultrason. Ferroelectr. Freq. Control 61, 1233 (2014).

[3] C. R. P. Courtney, C. E. M. Demore, H. Wu, A. Grinenko, P. D. Wilcox, S. Cochran, and B.W. Drinkwater, Appl. Phys. Lett. 104, 154103 (2014).

[4] B. T. Hefner and P. L. Marston, J. Acoust. Soc. Am. 106, 3313 (1999).

[5] J.-L. Thomas and R. Marchiano, Phys. Rev. Lett. 91, 244302 (2003).

[6] J. Lekner, J. Acoust. Soc. Am. 120, 3475 (2006).

[7] A. Anhäuser, R. Wunenburger, and E. Brasselet, Phys. Rev. Lett. 109, 034301 (2012).

[8] L. Zhang and P. L. Marston, J. Acoust. Soc. Am. 136, 2917 (2014).

[9] G. T. Silva, J. Acoust. Soc. Am. 136, 2405 (2014).

[10] K. D. Skeldon, C. Wilson, M. Edgar, and M. J. Padgett, New J. Phys. 10, 013018 (2008).

[11] K. Volke-Sepúlveda, A. O. Santillán, and R. R. Boullosa, Topologica 2, 016 (2009).
[12] C. E. M. Demore, Z. Yang, A. Volovick, S. Cochran, M. P. MacDonald, and G. C. Spalding, Phys. Rev. Lett. 108, 194301 (2012).

[13] Z. Y. Hong, J. Zhang, and B. W. Drinkwater, Phys. Rev. Lett. 114, 214301 (2015).

[14] P. L. Marston, J. Acoust. Soc. Am. 121, 3109 (2007).

[15] F. Mitri, IEEE Trans. Ultrason. Ferroelectr. Freq. Control 56, 1059 (2009).

[16] A. Marzo, S. A. Seah, B. W. Drinkwater, D. R. Sahoo, B. Long, and S. Subramanian, Nat. Commun. 6, 8661 (2015).

[17] D. Baresch, J.-L. Thomas, and R. Marchiano, Phys. Rev. Lett. 116, 024301 (2016).

[18] J. F. Nye and M. V. Berry, Proc. R. Soc. A 336, 165 (1974).

[19] S. Santesson and S. Nilsson, Anal. Bioanal. Chem. 378, 1704 (2004).

[20] L. Puskar, R. Tuckermann, T. Frosch, J. Popp, V. Ly, D. Mcnaughton, and B. R. Wood, Lab Chip 7, 1125 (2007).

[21] W. J. Xie, C. D. Cao, Y. J. Lü, Z. Y. Hong, and B. Wei, Appl. Phys. Lett. 89, 214102 (2006).

[22] M. Sundvik, H. J. Nieminen, A. Salmi, P. Panula, and E. Hæggström, Sci. Rep. 5, 13596 2015).

[23] A. M. Seddon, S. J. Richardson, K. Rastogi, T. S. Plivelic, A. M. Squires, and C. Pfrang, J. Phys. Chem. Lett. 7, 1341 (2016).

[24] C. G. Roessler et al., Structure 24, 631 (2016).

[25] K. A. Baldwin, S. L. Butler, and R. J. A. Hill, Sci. Rep. 5, 13596 (2015).

[26] D. Ahmed, A. Ozcelik, N. Bojanala, N. Nama, A. Upadhyay, Y. Chen, W. Hanna-Rose, and T. J. Huang, Nat. Commun. 7, 11085 (2016).

[27] D. Foresti and D. Poulikakos, Phys. Rev. Lett. 112, 024301 (2014).

[28] Z. Y. Hong, J. Zhang, and B. W. Drinkwater, Europhys. Lett. 110, 14002 (2015).

[29] J.-L. Thomas, T. Brunet, and F. Coulouvrat, Phys. Rev. E 81, 016601 (2010).

[30] M. Gecevičius, R. Drevinskas, M. Beresna, and P. G. Kazansky, Appl. Phys. Lett. 104, 231110 (2014).

[31] See Supplemental Material at http://link.aps.org/ supplemental/10.1103/PhysRevLett.120.044301 for further details on experiments, calculations, and movies, which includes Refs. [32-43].

[32] H. T. Oneil, J. Acoust. Soc. Am. 21, 516 (1949).

[33] J. J. Faran, J. Acoust. Soc. Am. 23, 405 (1951).

[34] P. J. Westervelt, J. Acoust. Soc. Am. 29, 199 (1957).

[35] G. G. Stokes, Reprinted in: Mathematical and Physical Papers, Vol. 1II (Cambridge University Press, Cambridge, U.K., 1851).

[36] S. I. Rubinow and J. B. Keller, J. Fluid Mech. 11, 447 (1961).

[37] G. Kirchhoff, Vorlesungen uber mathematische Physik: Mechanik (B. G. Teubner, Leipzig, 1883).

[38] P. P. Brown and D. F. Lawler, Journal of environmental engineering 129, 222 (2003).

[39] B. Oesterlé and T. B. Dinh, Exp. Fluids 25, 16 (1998).

[40] N. Lukerchenko, Y. Kvurt, A. Kharlamov, Z. Chara, and P. Vlasak, J. Hydrol. Hydromech. 56, 88 (2008)

[41] O. Sawatzki and J. Zierep, Acta Mech. 9, 13 (1970).

[42] H. Bruus, Lab Chip 12, 1014 (2012). 
[43] S. D. Danilov and M. A. Mironov, J. Acoust. Soc. Am. 107, 143 (2000).

[44] D. Baresch, J.-L. Thomas, and R. Marchiano, J. Acoust. Soc. Am. 133, 25 (2013).

[45] N. R. Heckenberg, T. A. Nieminen, M. E. J. Friese, and H. Rubinsztein-Dunlop, in International Conference on Singular Optics (1998), https://www.spiedigitallibrary .org/conference-proceedings-of-spie/3487/1/Trappingmicroscopic-particles-with-singular-beams/10.1117/12 .317715 .short?SSO $=1$.

[46] J. Ng, Z. Lin, and C. T. Chan, Phys. Rev. Lett. 104, 103601 (2010).

[47] N. Wang, J. Chen, S. Liu, and Z. Lin, Phys. Rev. A 87, 063812 (2013).

[48] P. Glynne-Jones, R. J. Boltryk, N. R. Harris, A. W. Cranny, and M. Hill, Ultrasonics 50, 68 (2010).

[49] V. Bollen, D. J. Zartman, T. M. Marston, and P. L. Marston, Proc. Meet. Acoust. 19, 070075 (2013).

[50] C. Shi, M. Dubois, Y. Wang, and X. Zhang, Proc. Natl. Acad. Sci. U.S.A. 114, 7250 (2017).

[51] D. Baresch, Doctoral dissertation, Université Pierre et Marie Curie-Paris VI, 2014.

[52] K. H. Lam, H.-S. Hsu, Y. Li, C. Lee, A. Lin, Q. Zhou, E. S. Kim, and K. K. Shung, Biotechnol. Bioeng. 110, 881 (2013).
[53] J. Lee, S.-Y. Teh, A. Lee, H. H. Kim, C. Lee, and K. K. Shung, Appl. Phys. Lett. 95, 073701 (2009).

[54] S. Zhao and J. Wallaschek, Arch. Appl. Mech. 81, 123 (2011).

[55] M. A. B. Andrade, A. L. Bernassau, and J. C. Adamowski, Appl. Phys. Lett. 109, 044101 (2016).

[56] M. A. B. Andrade, F. T. A. Okina, A. L. Bernassau, and J. C. Adamowski, J. Acoust. Soc. Am. 141, 4148 (2017).

[57] D. Zang, K. Lin, L. Li, Z. Chen, X. Li, and X. Geng, Appl. Phys. Lett. 110, 121602 (2017).

[58] A. D. Maxwell, M. Bailey, B. W. Cunitz, M. Terzi, A. Nikolaeva, S. Tsysar, and O. A. Sapozhnikov, J. Acoust. Soc. Am. 139, 2040 (2016).

[59] D. Foresti, G. Sambatakakis, S. Bottan, and D. Poulikakos, Sci. Rep. 3, 3176 (2013).

[60] H. M. Hertz, J. Appl. Phys. 78, 4845 (1995).

[61] S. Santesson, J. Johansson, L. S. Taylor, I. Levander, S. Fox, M. Sepaniak, and S. Nilsson, Anal. Chem. 75, 2177 (2003).

[62] A. Riaud, J.-L. Thomas, M. Baudoin, and O. B. Matar, Phys. Rev. E 92, 063201 (2015).

[63] A. Riaud, M. Baudoin, O. B. Matar, L. Becerra, and J.-L. Thomas, Phys. Rev. Applied 7, 024007 (2017).

[64] B. J. Mcmorran, A. Agrawal, I. M. Anderson, A. A. Herzing, H. J. Lezec, J. J. Mcclelland, and J. Unguris, Science 331, 192 (2011). 